\title{
Strain Elastography: A Valuable Additional Method to BI-RADS?
}

\section{Strain Elastografie: Eine wertvolle Zusatzmethode für BI-RADS?}

Authors

Xin-Bao Zhao ${ }^{1}$, Ji-Yi Yao ${ }^{1}$, Xin Chuan Zhou ${ }^{1}$, Shao-Yun Hao ${ }^{1}$, Wen Jie Mu², Lu-Jing Li ${ }^{1}$, Wen-Jing Zhong ${ }^{1}$, Zhi Hui ${ }^{1}$

Affiliation

1 Department of Ultrasound, Sun Yat-sen Memorial Hospital, Sun Yat-sen University, Guangzhou, Guangdong Province, China

2 Department of Ultrasound, Second Affiliated Hospital of Guangzhou Medical College, Guang Zhou, China

Key words

breast radiography, strain elastography, BI-RADS, breast cancer, strain ratio

received 14.08.2016

accepted 05.06.2017

Bibliography

DOI https://doi.org/10.1055/s-0043-115108

Published online: September 3, 2018 | Ultraschall in Med 2018; 39: 526-534 @ Georg Thieme Verlag KG, Stuttgart .

New York, ISSN 0172-4614

\section{Correspondence}

Dr. Zhi Hui

Department of Ultrasound, Sun Yat-sen Memorial Hospital, Sun Yat-sen University, Dept of Ultrasound, Sun Yat-sen Memorial Hospital of Sun Yat-sen University 107, 107 Yanjiangxi Road, 510120 Guangzhou, China Tel.: ++86/20/81332516

zhihui181@126.com

\section{ABSTRACT}

Background Breast lesions classified as BI-RADS-US 3 are probably benign and observation was recommended, while a considerable number of BI-RADS-US 4 lesions were benign, resulting in excessive biopsies. We focus exclusively on BI-RADS-US 3 and 4 lesions and hypothesize that improved diagnostic performance can be achieved by integrating realtime elastography (strain ratio) into the BI-RADS-US classification system.

Method From April 2010 to September 2015, 1071 lesions were included in the final analysis. After the conventional ultrasound examination, the BI-RADS-US (2013) classification was used to evaluate the lesions. Then the strain ratios were calculated, and the final diagnosis was made on the basis of histological results. The sensitivity, specificity, accuracy, PPV and NPV were calculated and the AUCs were compared. Additionally, an analysis of the diagnostic performance expressed by the pretest and posttest probability of disease (POD) was performed in BI-RADS-US 3 and 4 A lesions.

Results With the cutoff point of 2.98, the sensitivity, specificity and accuracy of the strain ratio method were $86.9 \%$, $86.6 \%$ and $82.6 \%$, respectively. In BI-RADS-US 3 lesions, a suspicious strain ratio significantly modified the POD from $1.3 \%$ to a posttest POD of $29.8 \%$. In BI-RADS-US 4A lesions, a suspicious strain ratio significantly modified the POD from $8.5 \%$ to a posttest POD of $48.7 \%$.

Conclusion Ultrasonographic elastography (strain ratio) yields additional diagnostic information in the evaluation of BI-RADS-US 3 and 4 breast lesions. The strain ratios should be integrated into the BI-RADS-US classification system and into daily practice.

\section{ZUSAMMENFASSUNG}

Hintergrund Herdbefunde der Brust, die mit BI-RADS-US Kategorie 3 bewertet wurden, sind vermutlich gutartig und eine Kontrolle wurde empfohlen, während solche mit BI-RADS-US Kategorie 4 in beträchtlichem Maße gutartig waren, was zu übermäßigen Biopsien führte. Unser Fokus gilt ausschließlich den Herdbefunden der BI-RADS-US Kategorie 3 und 4 und wir vermuten, dass die diagnostische Leistung durch Aufnahme der Echtzeit-Elastografie (Strain-Ratio) in die BI-RADS-US-Befund-Kategorien verbessert werden kann.

Methoden Von April 2010 bis September 2015 wurden 1071 Herdbefunde in die Endanalyse aufgenommen. Nach Durchführung der konventionellen Sonografie wurden die BI-RADSUS-Kategorien (2013) zur Bewertung der Herdbefunde angewandt. Dann wurden die Strain-Ratios berechnet, die Enddiagnose wurde histologisch gesichert. Sensitivität, Spezifität, Genauigkeit, PPW und NPW wurden berechnet und die AUCs wurden verglichen. Zusätzlich wurde bei den BI-RADSUS 3 und 4A Herdbefunden eine Analyse der diagnostischen Leistung mittels Prätest- und Posttest-POD („,probability of disease“) durchgeführt.

Ergebnis Bei einem Grenzwert von 2,98 erzielte die StrainRatio-Methode eine Sensitivität von $86,9 \%$, eine Spezifität von 86,6\% und eine Genauigkeit von 82,6\%. Bei BI-RADS-US 3-Herdbefunden führte eine auffällige Strain-Ratio zu einer signifikanten Veränderung der POD von $1,3 \%$ auf $29,8 \%$ 
(Posttest). Bei BI-RADS-US 4A-Herdbefunden veränderte eine auffällige Strain-Ratio die POD signifikant von $8,5 \%$ auf $48,7 \%$ (Posttest).

Schlussfolgerung Die Ultraschall-Elastografie (Strain-Ratio) liefert zusätzliche diagnostische Informationen bei der Bewer- tung von Herdbefunden der Brust bei BI-RADS-US Kategorien 3 und 4. Die Strain-Ratio sollte in die BI-RADS-US-BefundKategorien und in die tägliche Praxis aufgenommen werden.

\section{Introduction}

The most prevalent cancer in the world is breast cancer (4.4 million survivors up to 5 years after diagnosis) [1]. According to the last global data, about 400000 women died from breast cancer, representing 1.6 percent of all female deaths [2, 3]. Fortunately, breast cancer mortality is decreasing in many high-risk countries due to intensified early detection, resulting in the diagnosis of smaller, earlier stage tumors, and advances in treatment [4].

In earlier US studies, US diagnosis was made according to differences in the size, shape, and boundary of lesions [5]. In 2003, the standardized BI-RADS-US classification system was proposed by the American College of Radiology (ACR) [6]. Nevertheless, in our clinical observation, BI-RADS-US 3 and BI-RADS-US 4 lesions, especially BI-RADS-US 4A lesions, remain a critical category. In a prospective study by Barr RG, BI-RADS category 3 lesions have a low malignancy rate $(0.8 \%)$ and only $0.1 \%$ of the cancers had suspicious changes at 6 -month follow-up [7]. In a retrospective study, $3 \%$ of BI-RADS-US 3 lesions were diagnosed as malignant, leading to a delayed diagnosis of cancer in many patients [8], while a considerable percentage of BI-RADS-US 4A lesions was confirmed to be benign, resulting in excessive biopsies. Therefore, a suitable diagnostic predictor of malignancy should be explored in BI-RADS-US 3 and BI-RADS-US 4A lesions.

In the last decade, strain elastography (SE) was found to be an efficient examination method for the diagnosis of liver lesions, thyroid cancer and breast cancer [9, 10]. In 2013, the revised BI-RADS-US classification system indicated stiffness, as a feature of malignant masses, should be integrated into breast tumor diagnosis [11]. After Waki et al. presented a new diagnosis system using the strain ratio measurement [12], the stiffness of breast lesions could be semi-quantitatively calculated with the same depth of breast tissue as the reference and the subjective bias could be avoided [13].

Therefore, with a focus on BI-RADS-US 3 and BI-RADS-US 4 lesions, our retrospective study scrutinized whether the strain ratio system can filter out malignancy in BI-RADS-US 4 lesions and identify malignancy in BI-RADS-US 3 lesions. The aim of this study was to assess whether the strain ratio could be used as a further characterization of lesions that are initially categorized as BI-RADS-US 3 or BI-RADS-US 4 and whether the strain ratio should be integrated into the BI-RADS-US classification system for precise prediction of malignancy.

\section{Methods}

\section{General design}

This study was carried out at the Sun Yat-sen Memorial Hospital. Data was collected from April 2010 to September 2015. All patients were examined with B-mode sonography and strain elastography. As we were using a standard of care clinical protocol, the responsible ethics committee did not require additional approval for the non-interventional design of our retrospective study. We obtained consent from each study patient prior to data collection.

\section{Patients}

Patients with a sonographically visible lesion classified as BI-RADSUS 2 - 5 were regarded as being suitable for our study. Any patients with radiotherapy and chemotherapy of breast cancer, skin disorders, inflammatory conditions of the breast, a distance between the front boundary of the lesion and the skin of $>4 \mathrm{~cm}$ were excluded. A total of 1301 lesions in 1080 patients were tested. Of these, 1071 lesions in 976 patients were included in the final analysis ( $\triangleright$ Fig. 1). Histological confirmation was performed in all cases.

\section{B-mode sonography and strain elastography examinations}

Both the B-mode sonography and strain elastography examinations were performed by 3 radiologists with more than 5 years of experience in US and SE. Two ultrasound scanners were used: Hitachi EUB-900 and Hitachi Hi-Vision PREIRUS (Hitachi Medical Corporation, Inc., Tokyo, Japan) equipped with the EUP L74 M linear transducer $(5-13 \mathrm{MHz}, 5.0 \mathrm{~cm})$ or the EUP-L65 linear probe $(6-14 \mathrm{MHz}, 3.8 \mathrm{~cm})$.

Firstly, all patients underwent bilateral whole-breast ultrasound examination. The BIRADS system [11] (BI-RADS category 2: benign; BI-RADS category 3: probably benign; BI-RADS category 4a: low suspicion; BI-RADS category 4b: intermediate suspicion; BI-RADS category 4c: moderate suspicion; BI-RADS category 5: highly suggestive of malignancy) was used to obtain a summative evaluation of the US features of each lesion.

Secondly, on real-time SE, the elastogram box mostly extended from the subcutaneous fat layer to the greater pectoral muscle to scan sufficient breast tissue. To obtain an optimal elasticity image, the process was repeated when the pressure indicator bar displayed an index of 3-4 until a stable image was acquired. The SE image was illustrated on a color map, with red, blue and green indicating soft, intermediate and hard elasticity, respectively. The strain ratio measurement was performed with a same-depth area as an internal reference [13].The region of interest (ROI) including 


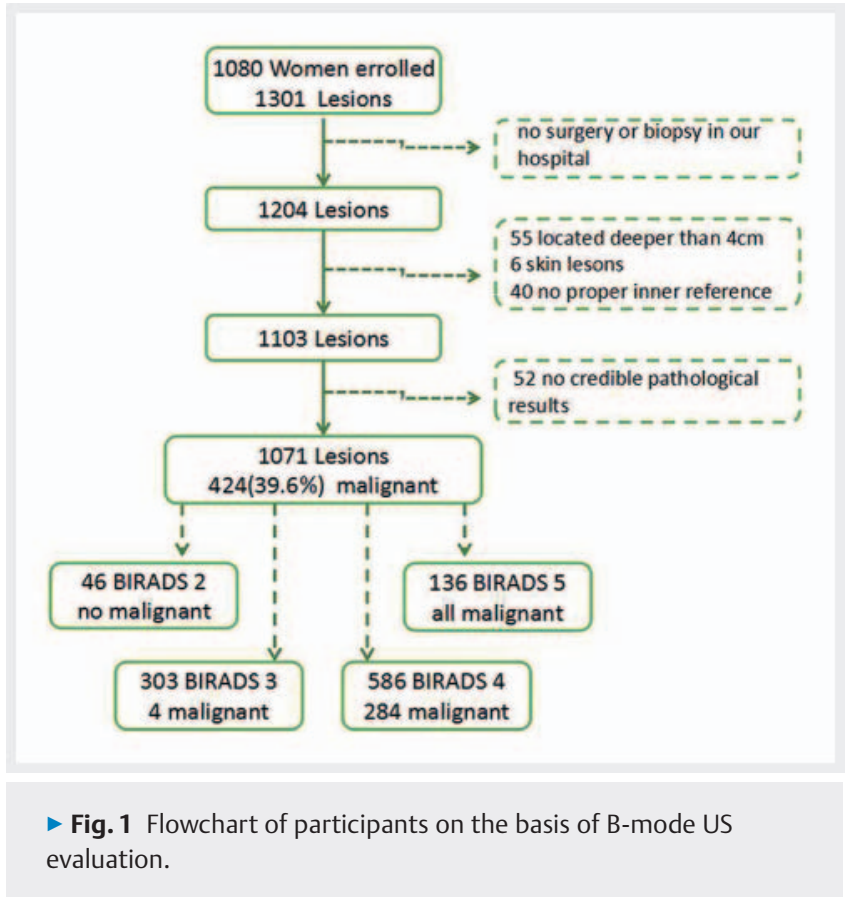

the lesion was expressed as A, and another ROI including the same-depth area was expressed as B. The strain ratio was automatically calculated as a B/A ratio.

Further diagnostic steps (mammography, core needle biopsy, open biopsy or follow-up examinations) were conducted. The histology of all of the lesions was established with an ultrasoundguided, 14-gauge, automated gun core biopsy, or an open biopsy. Only biopsy results with a definitive diagnosis were accepted.

\section{Statistical Analysis}

Microsoft Office Excel 2003 (Microsoft Corporation) was used for data collection. Statistical analysis was performed using SPSS version 13 software (SSPS Inc, Chicago, IL) and MedCalc (MedCalc Software, Mariakerke, Belgium).

The best cutoff point of strain ratio was obtained by calculating the Youden's index. Receiver operating characteristic curves (ROCs) were used to evaluate the diagnostic efficiency. The z-test was then used to compare the area under the curve (AUC). The accuracy, sensitivity, and specificity were compared by McNemar's test. The Mann-Whitney $U$ test was used to compare the medians of two independent groups for continuous data. The Fisher's exact test was used to compare independent groups for categorical variables.

With a focus on BI-RADS-US 3 and 4 lesions, we calculated the pretest probability of disease (POD) as well as the posttest POD in the high-risk group and in the low-risk group. Pairwise comparisons were performed using the $z$-test. Statistical significance was assumed at $\mathrm{P}<0.05$ for all tests.

\section{Results}

1071 lesions in 976 patients were included in the final analysis ( $\triangleright$ Fig. 1). The patients' age, lesion size and strain ratio were com- pared between benign and malignant subgroups ( $\downarrow$ Table 1 ).

- Table 2 shows the histological diagnosis of the lesions.

The diagnostic performance of BI-RADS-US was calculated: the overall sensitivity of B-mode US was $99.0 \%$ (420 of 424), with a $95 \%$ confidence interval $(\mathrm{Cl})$ of $95.1 \%$ to $99.3 \%$; the specificity was $53.3 \%$ (345 of 647 ), with a $95 \% \mathrm{Cl}$ of $54.2 \%$ to $60.9 \%$; and the accuracy was $71.4 \%$ (765 of 1071 ), with a $95 \% \mathrm{Cl}$ of $68.6 \%$ to $74.8 \%$. The area under the receiver operating characteristic curve (AUC) was 0.985 (95\% Cl: $0.970,0.991)$ for BI-RADS assessment alone.

\section{Strain ratio of breast lesions}

The mean strain ratio of the 647 benign lesions was $1.97 \pm 1.24$, ranging from 0.34 to 8.12 . The 1 st quartile, median and 3 rd quartile strain ratios were $1.22,1.63,2.41$, respectively. The mean strain ratio of the 424 malignant lesions was $7.97 \pm 4.53$, ranging from 0.77 to 56.70 . The 1 st quartile, median, and 3 rd quartile strain ratios were $3.64,5.41,9.24$, respectively. The strain ratios for malignant lesions were higher than for benign ones $(\mathrm{P}<0.001)$.

The ROC curve was used to assess the diagnosis performance of the strain ratio method. The AUC was 0.936 , and the maximum Youden's index was 0.76 . As the best cutoff point is defined when the maximum Youden's index value is attained, the best cutoff point in this study was 2.98. With this best cutoff point, the sensitivity, specificity, accuracy, PPV and NPV of the strain ratio method were $86.9 \%, 86.6 \%, 82.6 \%, 78.8 \%$, and $92.0 \%$, respectively.

\section{Analysis of the strain ratio distribution of each BI- RADS-US category lesion ( $\vee$ Table 3 )}

46 lesions were classified as BI-RADS-US 2. According to histology, all (100\%) of the 46 lesions were benign. The mean strain ratio of BI-RADS-US 2 lesions was $1.20 \pm 0.46$. The stain ratio of all BI-RADS-US 2 lesions were under 2.98.

303 lesions were classified as BI-RADS-US 3. According to histology, $98.7 \%$ (299 of 303) were benign. The mean strain ratio was $1.75 \pm 0.79$. Among the 299 benign lesions, 7 lesions had a strain ratio higher than 2.98 . While $1.3 \%$ (4 of 303) of these lesions were malignant, the mean strain ratio was $3.86 \pm 1.02$. Among the 4 malignant lesions, 3 lesions had a strain ratio higher than 2.98 .

586 lesions were classified as BI-RADS-US 4. According to histology, $51.2 \%$ (300 of 586) of these lesions were benign. The mean strain ratio was $2.39 \pm 1.22$. Among the 300 benign lesions, 96 lesions had strain ratios higher than 2.98. While $48.8 \%$ (286 of 586 ) of the lesions were malignant, the mean strain ratio was $7.06 \pm 4.25$. Among the 286 malignant lesions, 41 lesions had strain ratios lower than 2.98 . Further analysis of these lesions is in the next part.

136 lesions were classified as BI-RADS-US 5. According to histology, all (100\%) of the 136 lesions were malignant. The mean strain ratio was $10.19 \pm 8.97$. Among the 136 malignant lesions, 9 lesions had a strain ratio lower than 2.98, including 7 IDC and 2 DCIS lesions. 
- Table 1 Participant characteristics of 976 patients with 1071 breast lesions.

\begin{tabular}{|c|c|c|c|c|}
\hline characteristic & $\begin{array}{l}\text { malignant } \\
n=424 \text { (39.6\%) }\end{array}$ & $\begin{array}{l}\text { benign } \\
n=647(60.4 \%)\end{array}$ & p-value & $\begin{array}{l}\text { all lesions } \\
n=1071\end{array}$ \\
\hline \multicolumn{5}{|l|}{ age (y) } \\
\hline median $^{1}$ & $49(14-88)$ & $41(17-81)$ & $<0.001$ & $46.5(14-88)$ \\
\hline mean $^{2}$ & $50.7 \pm 12.0$ & $41.6 \pm 10.3$ & $<0.001$ & $45.8 \pm 14.7$ \\
\hline \multicolumn{5}{|c|}{ lesion diameter (mm) } \\
\hline median $^{1}$ & $12.0(3.2-55)$ & $9.1(4.6-47.5)$ & $<0.001$ & $11.6(3.2-55)$ \\
\hline mean $^{2}$ & $13.5 \pm 9.0$ & $11.2 \pm 7.7$ & $<0.001$ & $12.9 \pm 8.4$ \\
\hline \multicolumn{5}{|l|}{ strain ratio } \\
\hline median $^{1}$ & $5.41(0.77-56.70)$ & $1.63(0.34-8.12)$ & $<0.001$ & $2.87(0.34-56.70)$ \\
\hline mean $^{2}$ & $7.97 \pm 4.53$ & $1.97 \pm 1.24$ & $<0.001$ & $4.53 \pm 3.92$ \\
\hline
\end{tabular}

- Table 2 Histological diagnosis of 976 patients with 1071 breast lesions.

\begin{tabular}{|l|r|l|l|}
\hline benign lesions (n= 647) & $\mathbf{n}$ & malignant lesions $\mathbf{( n = 4 2 4 )}$ & $\mathbf{n}$ \\
\hline fibroadenoma & 406 & invasive ductal carcinoma & 329 \\
\hline fibroadenomatous hyperplasia & 72 & ductal carcinoma in situ & 34 \\
\hline papilloma & 62 & invasive lobular carcinoma & 11 \\
\hline adenopathy & 34 & mucinous carcinoma & 9 \\
\hline phyllodes tumor & 29 & invasive papillocarcinoma \\
\hline chronic inflammation & 17 & neuroendocrine carcinoma & 7 \\
\hline atypical ductal hyperplasia & 5 & intraductal papillary carcinoma \\
\hline tubular adenoma & 5 & invasive adenocarcinoma \\
\hline juvenile hyaline fibromatosis & 4 & medullary carcinoma \\
\hline lipoma & 3 & lymphoma \\
\hline other benign lesions & 10 & other malignant lesions \\
\hline
\end{tabular}

\section{Analysis of the strain ratio method to further evaluate BI-RADS-US 3 and BI-RADS-US 4 lesions}

The 4 malignant BI-RADS-US 3 lesions included 3 IDC lesions (strain ratios: $3.18,4.88,4.59$ ) and 1 DCIS lesion (strain ratio: 2.82). All 4 malignant lesions had higher strain ratios than the 1st quartile, median and the $3 \mathrm{rd}$ quartile strain ratios of all benign lesions (1.29, 1.68, and 2.41, respectively). According to the ROC curve, the AUC of the strain ratio method in BI-RADS-US 3 lesions was 0.977 ( $\triangleright$ Fig. 2).

199 lesions were classified as BI-RADS-US 4A, $91.5 \%$ (182 of 199 ) of which were benign. The mean strain ratio was 1.75 . While $8.5 \%$ (17 of 199) of these lesions were malignant, the mean strain ratio was 5.13. Among these malignant lesions, all 17 lesions had higher strain ratios than 1.29 and 1.63 , the 1 st quartile and the median strain ratios of all benign lesions, respectively. 16 had higher strain ratios than 2.41 , the 3 rd quartile strain ratio of all benign lesions. According to the ROC curve, the AUC of the strain ratio method in BI-RADS-US 4A lesions was 0.929 ( $\bullet$ Fig. 3 ).

177 lesions were classified as BI-RADS-US 4B, 54.2\% (96 of 177 ) of which were benign. The mean strain ratio was 3.94 . While $45.8 \%$ (81 of 177 ) of these lesions were malignant, the mean strain ratio was 7.14. According to the ROC curve, the AUC of the strain ratio method in BI-RADS-US 4B lesions was 0.740 ( $\triangleright$ Fig. 4).

210 lesions were classified as BI-RADS-US 4C, 10.4\% (22 of 210 ) of which were benign. The mean strain ratio was 4.57 . While $89.6 \%$ (188 of 210 ) of these lesions were malignant, the mean strain ratio was 7.72. According to the ROC curve, the AUC of the strain ratio method in BI-RADS-US 4C lesions was 0.710 ( $>$ Fig. 5). 
- Table 3 Strain ratio distribution of each BI-RADS-US category lesion.

\begin{tabular}{|c|c|c|c|c|c|c|c|c|c|c|}
\hline \multirow{2}{*}{$\begin{array}{l}\text { BIRADS } \\
\text { (n) }\end{array}$} & \multicolumn{2}{|l|}{ n } & \multicolumn{2}{|l|}{ B } & \multicolumn{2}{|l|}{ M } & \multicolumn{2}{|l|}{ B } & \multicolumn{2}{|l|}{ M } \\
\hline & ${ }^{1} B$ & ${ }^{2} M$ & $S R \geq 2.98$ & SR2.98 & $S R \geq 2.98$ & SR2.98 & mean & median & mean & median \\
\hline $2(46)$ & 46 & 0 & NA & 46 & NA & NA & $1.20 \pm 0.46$ & 1.23 & NA & NA \\
\hline $3(303)$ & 299 & 4 & 7 & 292 & 3 & 1 & $1.75 \pm 0.79$ & 1.63 & $13.86 \pm 1.02$ & 3.68 \\
\hline 4a (199) & 182 & 17 & 27 & 155 & 10 & 7 & $2.05 \pm 1.33$ & 1.65 & $25.13 \pm 4.99$ & 3.89 \\
\hline 4b (177) & 96 & 81 & 52 & 44 & 66 & 15 & $3.94 \pm 2.41$ & 3.21 & $37.51 \pm 7.14$ & 4.36 \\
\hline $4 c(210)$ & 22 & 188 & 17 & 5 & 169 & 19 & $4.57 \pm 2.39$ & 4.09 & ${ }^{4} 7.21 \pm 6.48$ & 5.39 \\
\hline $5(136)$ & 0 & 136 & NA & NA & 127 & 9 & NA & NA & $10.19 \pm 8.97$ & 6.76 \\
\hline \multicolumn{11}{|c|}{$\begin{array}{l}1 \text { B = benign. } \\
2 M=\text { malignant. } \\
{ }^{1} \text { Mean SR of malignant lesions of BI-RADS-3 was higher than that of benign ones }(P=0.002) \text {. } \\
{ }^{2} \text { Mean } \text { SR of malignant lesions of BI-RADS-4A was higher than that of benign ones }(P<0.001) \text {. } \\
{ }^{3} \text { Mean SR of malignant lesions of BI-RADS-4B was higher than that of benign ones }(P<0.001) \text {. } \\
{ }^{4} \text { Mean SR of malignant lesions of BI-RADS-4C was higher than that of benign ones }(P<0.001) \text {. }\end{array}$} \\
\hline
\end{tabular}

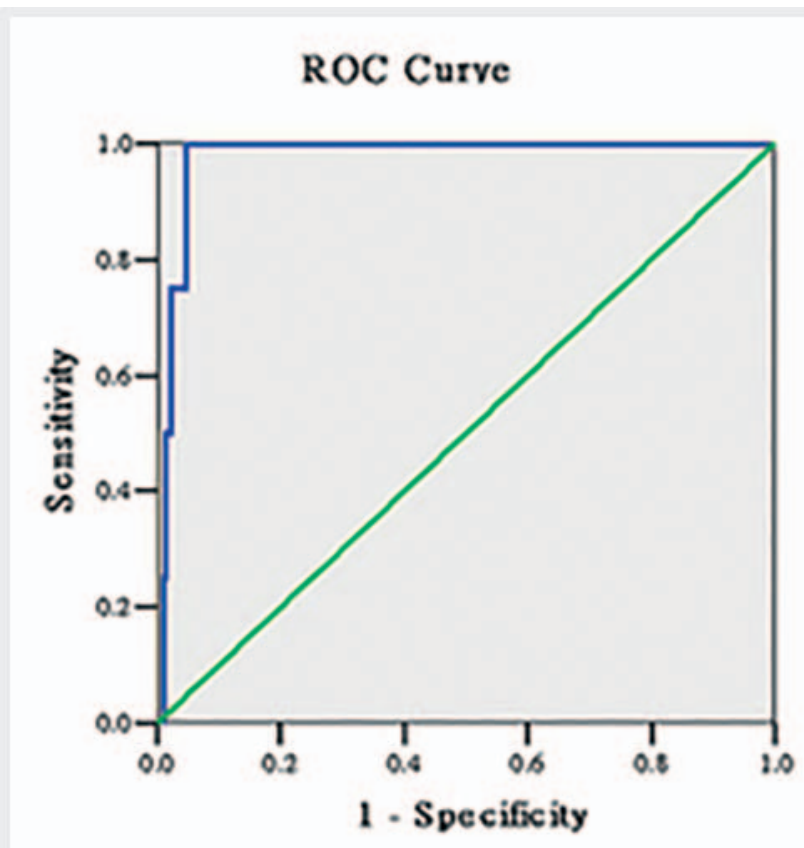

- Fig. 2 Receiver operating characteristic (ROC) curves for the strain ratio method in BI-RADS-US 3 lesions.

\section{Probability of disease in BI-RADS-US category 3 and $4 \mathrm{~A}$ lesions}

The pretest probability of disease in BI-RADS-US 3 lesions was $1.3 \%$ and increased significantly to $29.8 \%$ with an abnormal strain ratio (higher than 2.98 ). The posttest probability of disease with a normal strain ratio (lower than 2.98) decreased significantly to $0.3 \%$.

The pretest probability of disease in BI-RADS-US 4A lesions was $8.5 \%$ and increased significantly to $48.7 \%$ with an abnormal strain

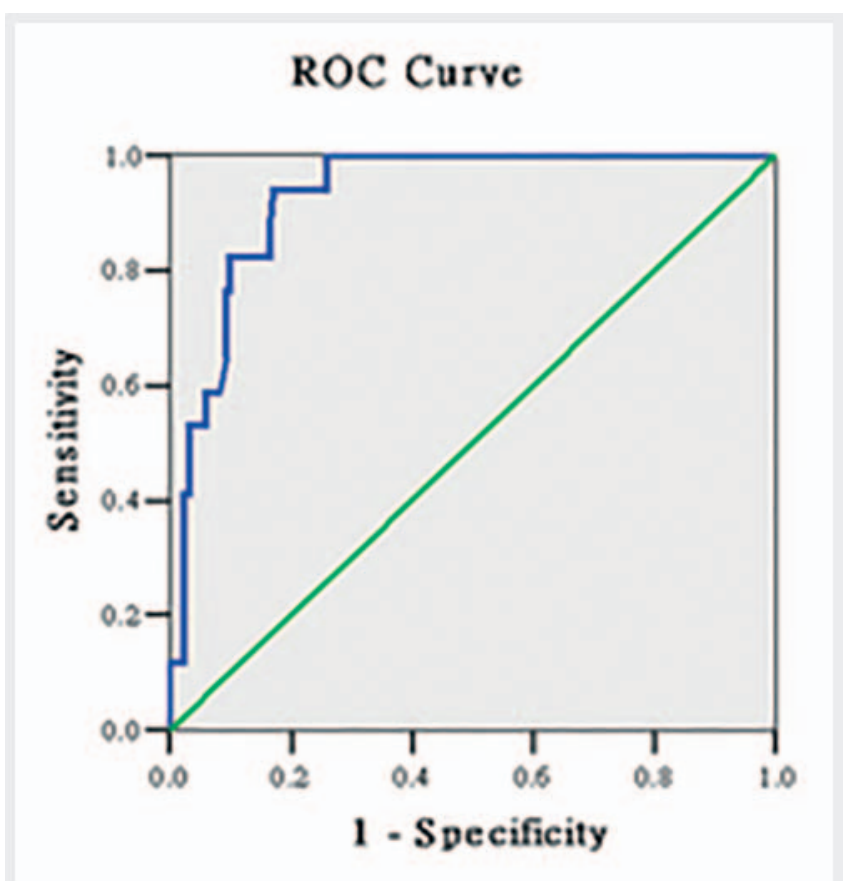

- Fig. 3 Receiver operating characteristic (ROC) curves for the strain ratio method in BI-RADS-US 4A lesions.

ratio (higher than 2.98). The posttest probability of disease with a normal strain ratio (lower than 2.98) decreased significantly to $4.5 \%$ ( Table 4).

\section{Effect of SE on sensitivity and specificity: BI-RADS-US 3 and $4 \mathrm{~A}$ lesions}

Inclusion of strain ratio for BI-RADS 3 lesions improved the overall sensitivity without a loss of specificity. Inclusion of strain ratio for BI-RADS 4A lesions improved the overall specificity without a loss 


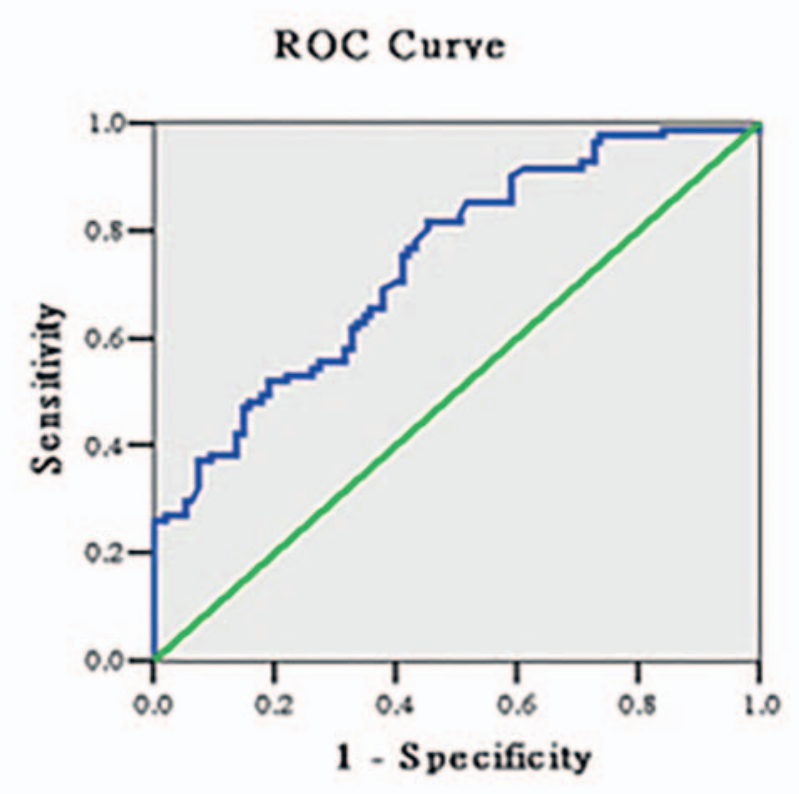

- Fig. 4 Receiver operating characteristic (ROC) curves for the strain ratio method in BI-RADS-US 4B lesions.

of sensitivity. By adding SE information to all lesions, the overall specificity improved from $53.3 \%$ for BI-RADS assessment alone to $76.4 \%$ ( $\triangleright$ Table 5 ). Two strategies were used for combining the BI-RADS category and strain ratio. The aggressive combination: the lesion was diagnosed as malignant when either SR or BIRADS yielded a positive result. The conservative combination: the lesion was diagnosed as malignant when both SR and BI-RADS yielded a positive result. The lesion was diagnosed as benign by both strategies only if both methods yielded a negative result. The aggressive combination provided better diagnostic performance than the conservative combination, with higher sensitivity in BI-RADS category 3 and higher specificity in 4 a lesions and all lesions.

\section{Discussion}

Two main forms of elastography have become established in clinical practice. However, other important implementations are to be expected as the field matures [14, 15]. The 2013 BI-RADS-US classification system stated that stiffness provides useful information that can be added to the diagnostic system. However, the combination of strain ratio and the BI-RADS-US classification system, especially with a focus on BI-RADS category 3 and category $4 a$, is relatively rare.

In clinical practice, SE examinations are usually performed after conventional ultrasound. The combination of these two methods is the most important point. Until now, there is minimal literature indicating any advantage from adding SE evaluation to lesions categorized BI-RADS-US $0,1,2$ or 5 . Our study is consistent with previous studies. However, improved diagnostic performance can be achieved for BI-RADS-US 3 and 4 lesions. Therefore, our study

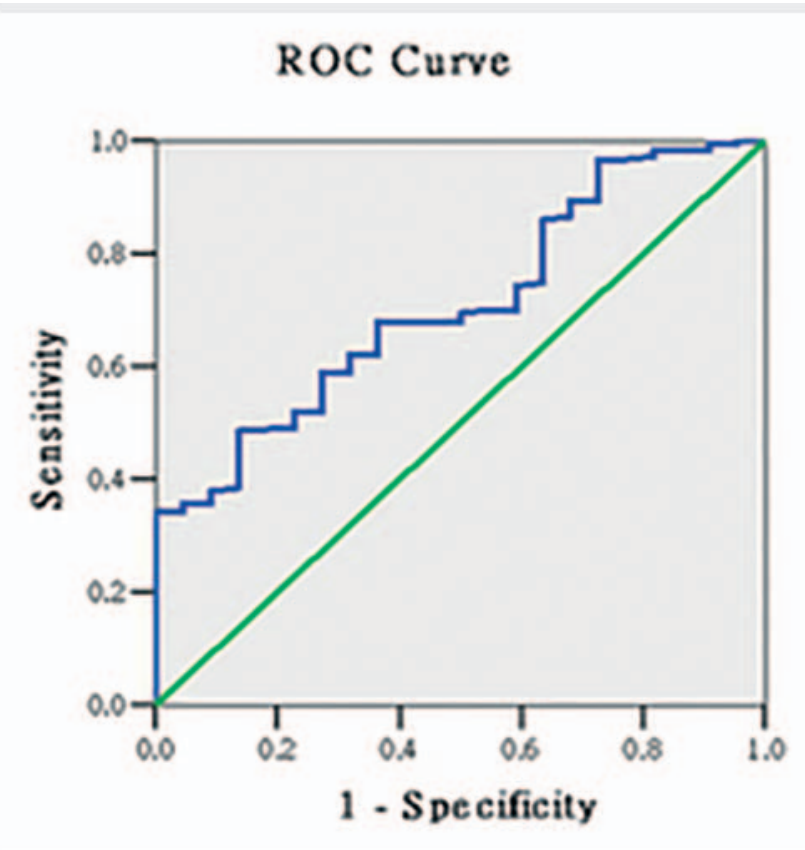

- Fig. 5 Receiver operating characteristic (ROC) curves for the strain ratio method in BI-RADS-US 4C lesions.

- Table 4 Probability of disease in BI-RADS-US 3 and BI-RADS-US 4A lesions after including strain ratio.

\begin{tabular}{|l|l|l|l|l|}
\hline \multicolumn{4}{|c|}{ BI-RADS 3 lesions } & \multicolumn{2}{|c|}{ BI-RADS 4A lesions } \\
\hline & SR $\geq 2.98$ & SR $<2.98$ & SR $\geq 2.98$ & SR $<2.98$ \\
\hline \\
\hline
\end{tabular}

was focused on breast lesions with an unclear diagnosis and requiring more advanced assessment.

In our study, with a cutoff point of 2.98 , the sensitivity and specificity of the strain ratio method were $86.9 \%$ and $86.6 \%$, respectively. This is comparable to previous studies. The sensitivity ranged from $73.9 \%$ to $92.4 \%$ and the specificity from $86.4 \%$ to $95.2 \%$ [16 - 20]. Adding SR to BI-RADS feature analysis improved the specificity of breast US lesion assessment without a loss of sensitivity. Interestingly, this result is not only consistent with 
- Table 5 Estimates of effect of combining SR with BI-RADS 3 and 4a lesions.

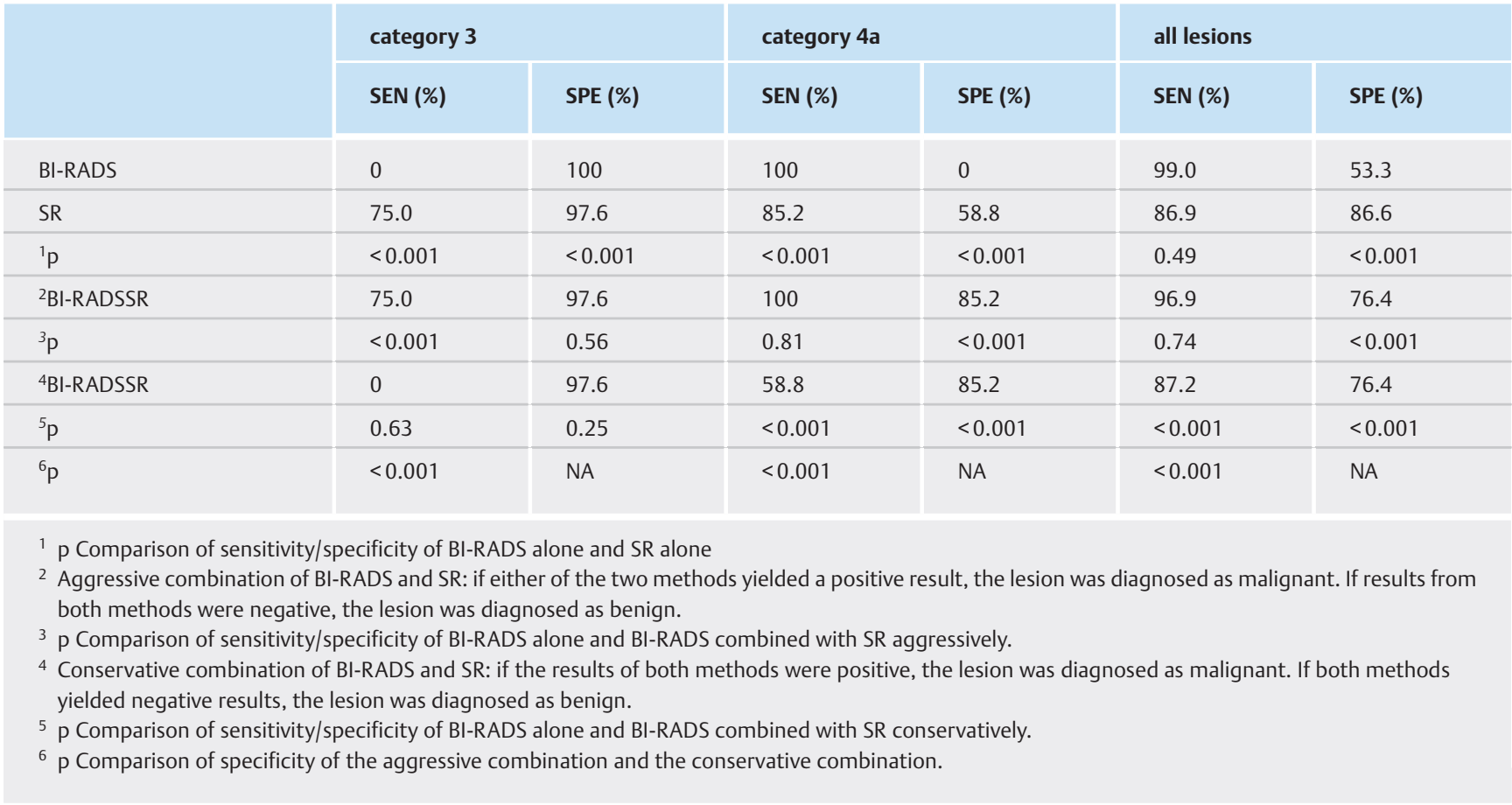

strain elastography but is also consistent with emerging shear wave elastography (SWE) technology [21].

In our study, the risk for malignancy in BI-RADS-US 3 lesions is $1.3 \%$, i. e., relatively low. With a suspicious strain ratio, the risk of malignancy increased significantly to a POD of $29.8 \%$. Usually BI-RADS-US 3 lesions only require follow-up. However, in light of the high posttest probability of malignancy, we encourage immediate biopsy of BI-RADS-US 3 lesions exhibiting a suspicious strain ratio ( $>$ Fig. 6 ).

BI-RADS-US 4 lesions cover a wide range of likelihood of malignancy (2 95\%). However, the majority of BI-RADS-US 4A lesions are in fact benign. Strain ratio allowed helpful categorization of lesions into a low-risk group (posttest POD $4.5 \%$ ) and a high-risk group (posttest POD $48.7 \%$ ). We suggest follow-up of BI-RADSUS 4A lesions with a normal strain ratio ( $\triangleright$ Fig. 7) and immediate biopsy of BI-RADS-US 4A lesions with a suspicious strain ratio ( $\triangleright$ Fig. 8). Biopsy in these cases could be expected to yield at least 1 carcinoma per 3 biopsies. Furthermore, in the BI-RADS-US 4A lesions in our study, all 17 malignant lesions had a higher strain ratio than 1.29 and 1.63 , the 1 st quartile and the median strain ratios of all benign lesions, respectively. If we choose the median strain ratio as the diagnostic criteria, the positive rate of biopsy will be improved because missed diagnoses will bring more serious consequences for patients.

However, we found that strain ratios did not provide beneficial information for BI-RADS-US 4B and 4C lesions. The 2013 BI-RADSUS classification system emphasized that elastography evaluation should not override the more predictive morphologic features of malignancy [11]. If a lesion was already highly suspicious on the mammogram and/or conventional ultrasound, the stiffness would not have an effect on the subsequent management of the patient.

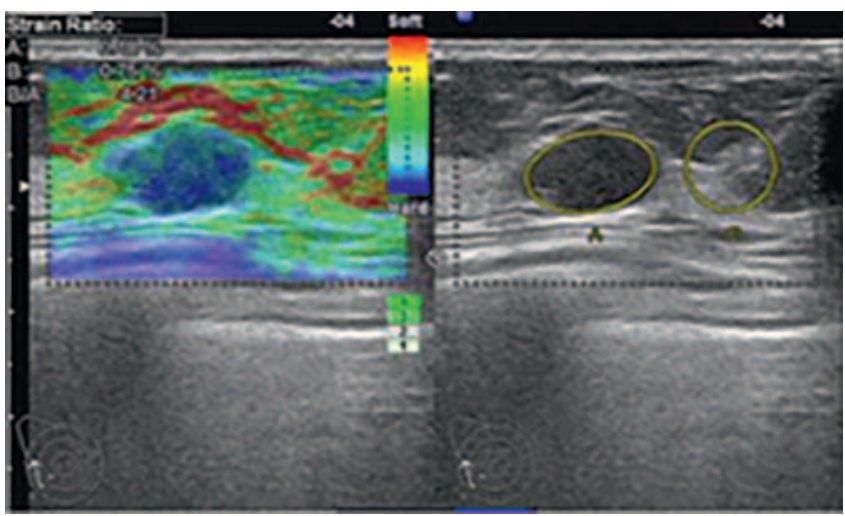

- Fig. 6 UE image of a neuroendocrine carcinoma in a 34-year-old woman. The lesion was classified as BI-RADS-US 3. The strain ratio was 4.21 .

Therefore, on account of the high probability of malignancy, we suggest that a biopsy should be performed in every BI-RADS-US $4 \mathrm{~B}$ or $4 \mathrm{C}$ lesion with a suspicious strain ratio.

Although the strain ratios of malignant lesions were significantly higher than those of benign ones, there is overlap in the elasticity coefficient for different tissues. Therefore, false-negative and false-positive results were unavoidable [22, 23]. Most false-negative lesions were invasive ductal carcinomas (IDCs) and ductal carcinomas in situ (DCISs). In our study, the majority of malignant lesions were IDCs. Only $10 \%$ (33 of 329) were misdiagnosed. DCIS has been shown to be softer than IDC ( $\triangleright$ Fig. 9), which is consistent with Thomas et al. [24]. Meanwhile, most false-positive lesions were intraductal papillomas (38 of 144 false-positive results). This is similar to Ann et al. [25], who sug- 

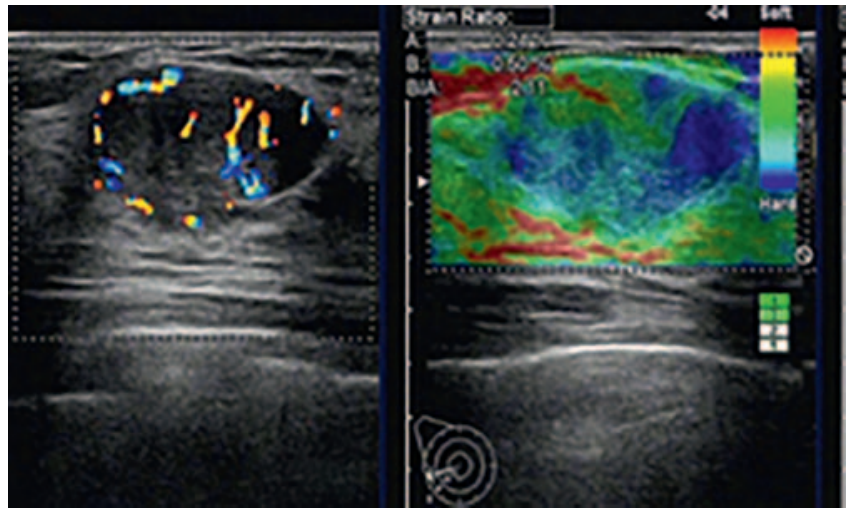

- Fig.7 UE image of a fibroadenoma in a 46-year-old woman. The lesion was classified as BI-RADS-US 4A. The strain ratio was 2.11 .

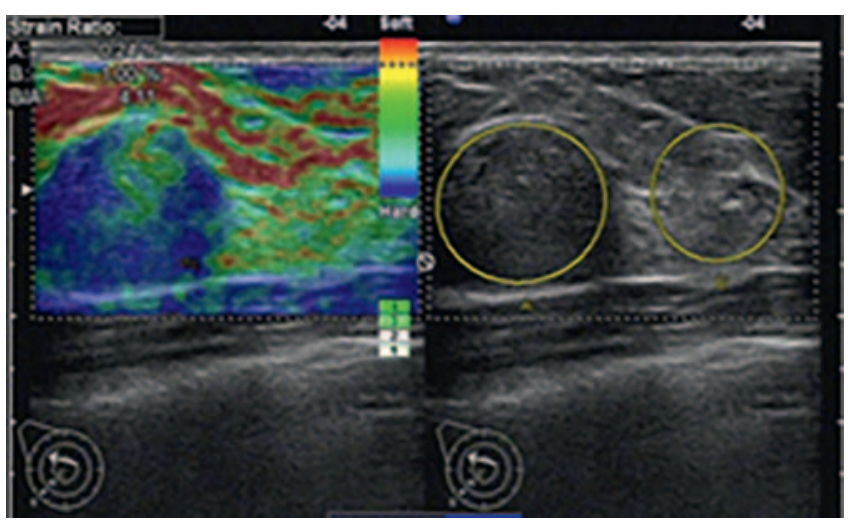

- Fig. 8 UE image of an IDC in a 43-year-old woman. The lesion was classified as BI-RADS-US 4A. The strain ratio was 4.11.

gested that the mean elasticity score of intraductal papillomas was higher than that of fibrocystic changes ( $\bullet$ Fig. 10). The second most common false-positive results were fibroadenomas (27 of 144 false-positive results), 3 of which were complicated by calcification and 5 of which were lager than $20 \mathrm{~mm}$.

In this study, the only malignant BIRADS-3 lesion misdiagnosed by SR with a strain ratio of 2.82 was a DCIS, while 5 of the 7 false-negative BIRADS 4a lesions misdiagnosed by SR were DCISs. 7 benign BIRADS-3 lesions had a strain ratio higher than 2.98, including 4 papillomas and 3 fibroadenomas ( 2 lesions lager than $30 \mathrm{~mm}$, 1 lesion with calcification). 27 false-positive BIRADS 4a lesions contained 12 papillomas (3 lesions with calcification), 7 fibroadenomas ( 2 lesions lager than $30 \mathrm{~mm}$, 4 lesions with calcification, 1 lesion with fibrous hyperplasia), 5 phyllodes tumors and 3 cases of chronic inflammation.

The main limitation of our study is the operator dependence. To a certain degree, this is inevitable in all studies of SE operation. Fortunately, all of the operators in our study were experienced. As a semi-quantitative method, strain ratio is more subjective and could avoid inter-operator variability. Another limitation is the lack of comparative studies. To prove our concept, a prospective, multi-center trial might be needed to fully evaluate the strain ratio method.

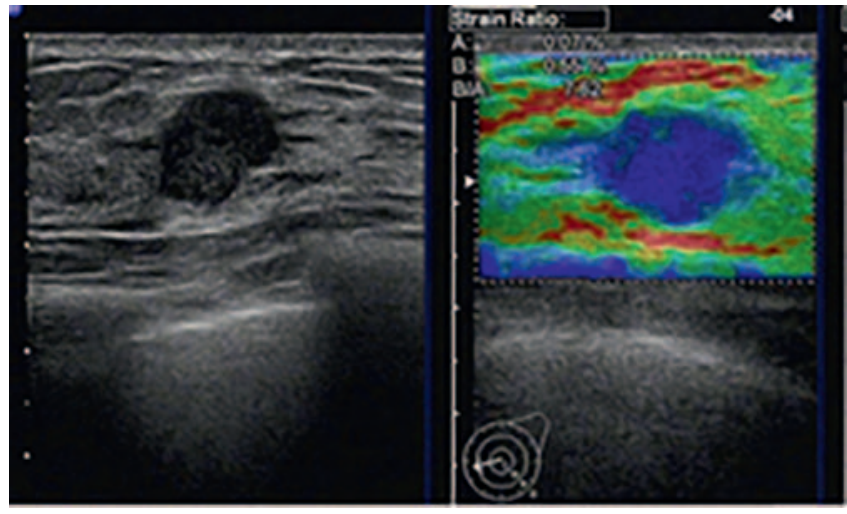

- Fig. 9 UE image of a papilloma in a 24-year-old woman. The lesion was classified as BI-RADS-US 3. The strain ratio was 7.62.

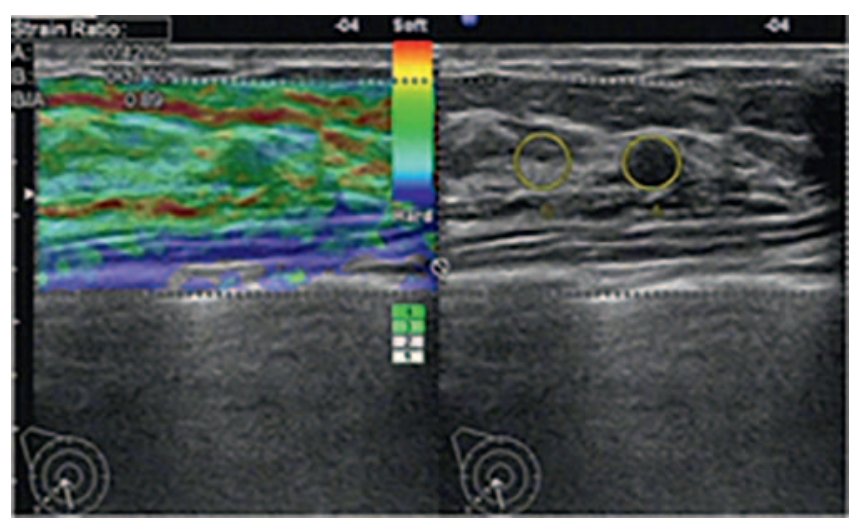

- Fig. 10 UE image of a DCIS in a 39-year-old woman. The lesion was classified as BI-RADS-US 2. The strain ratio was 0.89 .

\section{Conclusion}

In our study, using the best cutoff point of 2.98 , the stain ratio method successfully differentiated between benign and malignant breast masses. This semi-quantitative method not only improved the specificity of breast US lesion assessment without a loss of sensitivity but also yielded additional diagnostic information in the evaluation of BI-RADS-US 3 and 4 lesions. It should be integrated into the BI-RADS-US classification system and daily practice.

\section{Conflict of Interest}

The authors declare that they have no conflict of interest.

\section{Reference}

[1] Parkin DM, Bray F, Ferlay J et al. Global cancer statistics 2002. CA Cancer J Clin 2005; 55 (2): $74-108$

[2] IARC. Globocan Database. Lyon: International Agency for Research on Cancer. 2008

[3] IARC. World Cancer Report 2008. Lyon: International Agency for Research on Cancer, WHO Press. 2008 
[4] Parkin DM, Fernandez LM. Use of statistics to assess the global burden of breast cancer. Breast J 2006; 12 (Suppl. 1): S70 - S80

[5] Stavros AT, Thickman D, Rapp CL et al. Solid breast nodules: use of sonography to distinguish between benign and malignant lesions. Radiology 1995; 196: $123-134$

[6] American College of Radiology. Illustrated breast imaging reporting and data system (BI-RADS)-ultrasound. 1st ed. Reston. VA: American College of Radiology 2003

[7] Barr RG, Zhang Z, Cormack JB et al. Probably benign lesions at screening breast US in a population with elevated risk: prevalence and rate of malignancy in the ACRIN 6666 trial. Radiology 2013; 269 (3): 701 - 712

[8] Hille H, Vetter M, Hackeloer B]. The accuracy of BI-RADS classification of breast ultrasound as a first-line imaging method. Ultraschall in Med 2012; 33 (2): $160-163$

[9] Tatsumi C, Kudo M, Ueshima K et al. Noninvasive evaluation of hepatic fibrosis using serum fibrotic markers, transient elastography (FibroScan) and real-time tissue elastography. Intervirology 2008; 51: 27 - 33

[10] Asteria C, Giovanardi A, Pizzocaro A et al. US-elastography in the differential diagnosis of benign and malignant thyroid nodules. Thyroid 2008; 18: $523-531$

[11] D’Orsi C], Sickles EA, Mendelson EB et al. ACR BI-RADS atlas, Breast Imaging Reporting and Data System. Reston, VA: American College of Radiology 2013

[12] Waki K, Murayama N, Matsumura T et al. Investigation of strain ratio using ultrasound elastography technique. Proceedings of ISICE 2007: $449-452$

[13] Zhi H, Xiao XY, Yang HY et al. Semi-quantitating stiffness of breast solid lesions in ultrasonic elastography. Acad Radiol 2008; 15: 1347 - 1353

[14] Au FW, Ghai S, Moshonov H et al. Diagnostic performance of quantitive shear wave elastography in the evaluation of solid breast masses:determination of the most discriminatory parameter. Am J Roentgenol 2014; 203 (3): W328-W336
[15] Zhi H, Xiao XY, Ou B et al. Could ultrasonic elastography help the diagnosis of small $(<1=2 \mathrm{~cm})$ breast cancer with the usage of sonographic BI-RADS classification? Eur J Radiol 2012; 81 (11): 3216-3221

[16] Itoh A, Ueno E, Tohno E et al. Breast disease: clinical application of US elastography for diagnosis. Radiology 2006; 239 (2): 341 - 350

[17] Tardivon A, El KhouryC, Thibault F et al. Elastography of the breast: a prospective study of 122 lesions. Journal de Radiologie 2007; 88 (5): $657-662$

[18] Zhi H, Xiao XY, Yang HY et al. Ultrasonic elastography in breast cancer diagnosis: strain ratio vs 5-point scale. Acad Radiol 2010; 17 (10): $1227-1233$

[19] Houelleu DemayM L, Monghal C, Bertrand P. An assessment of the performance of elastography for the investigation of BI-RADS 4 and BI-RADS 5 breast lesions: correlations with pathological anatomy findings. Diagn Interv Imaging 2012; 93 (10): 757 - 766

[20] Farrokh A, Wojcinski S, Degenhardt F et al. Diagnostic value of strain ratio measurement in the differentiation of malignant and benign breast lesions. Ultraschall in Med 2011; 32 (4): 400-405

[21] Berg WA, Cosgrove DO, Doré C] et al. Shear-wave elastography improves the specificity of breast US: the BE1 multinational study of 939 masses. Radiology 2012; 262 (2): 435 - 449

[22] Stachs A, Hartmann S, Stubert ] et al. Differentiating between malignant and benign breast masses: factors limiting sonoelastographic strain ratio. Ultraschall in Med 34 (2): 131 -136

[23] Ciurea AI, Bolboaca SD, Ciortea CA et al. The influence of technical factors on sonoelastographic assessment of solid breast nodules. Ultraschall in Med 2011; 32 (Suppl. 1): S27-S34

[24] Thomas A, Fischer T, Frey $\mathrm{H}$ et al. Real-time elastography - an advanced method of ultrasound: first results in 108 patients with breast lesions. Ultrasound Obstet Gynecol 2006; 28 (3): 335-340

[25] Yi A, Cho N, Chang JM et al. Sonoelastography for 1786 non-papable breast masses:diagnostic value in the decisionto biopsy. Eur Radio 2012; 22 (5): $1033-1040$ 